\title{
A comparative study of simple and choice reaction time in Parkinson's, Huntington's and cerebellar disease
}

\author{
Marjan Jahanshahi, Richard G Brown, C David Marsden
}

\begin{abstract}
The aim of the study was to compare the quantitative and qualitative similarities and differences in the performance of patients with Parkinson's disease, Huntington's disease and cerebellar disease on a number of reaction time tasks. Simple reaction time (SRT), uncued and fully cued four choice (CRT) tasks were performed by eight patients with Parkinson's disease after withdrawal of dopaminergic medication for an average of 14.4 hours; by seven non-demented patients with Huntington's disease and by eight patients with cerebellar disease.
\end{abstract} An S1 (warning signal/precue)-S2 (imperative stimulus) paradigm was used in all tasks, with the S1-S2 interval randomly varying between $0,200,800$, 1600 and $3200 \mathrm{~ms}$ across trials. The patients with Huntington's disease had a significantly longer SRT than those with Parkinson's disease. None of the other group differences in uncued and unwarned SRT and CRT was significant. For the patients with Parkinson's disease and those with cerebellar disease, unwarned SRT was faster than uncued and unwarned CRT. For the patients with Huntington's disease, this CRT/SRT difference was not significant. A warning signal before the imperative stimulus resulted in a reduction of reaction time in all three groups. Advance information provided by $S 1$ about the response that would be required by $S 2$ was used by patients in all three groups, evident from reaction times in the fully cued CRT task being faster than those in the uncued CRT condition. Patients with cerebellar disease had slower movement times in the SRT and CRT conditions compared with the patients with Parkinson's disease and Huntington's disease, whose times did not differ. In one SRT condition, when the absence of a warning signal was predictable, patients with cerebellar disease, and to a lesser extent those with Huntington's disease, were able to maintain a general motor readiness before the imperative stimulus. This was not the case for the patients with Parkinson's disease who seemed more dependent on the presence of a warning signal to reduce their reaction time. With a few exceptions, the pattern of results of the three groups were qualitatively similar. It may be concluded that similar reaction time deficits are found in Parkinson's disease, in patients with other disorders of the basal ganglia (Huntington's disease), as well as those with a disease sparing the basal ganglia (cerebellar disease). The non-specific slowness observed at the behavioural level may, however, have diverse central mechanisms.

\section{(F Neurol Neurosurg Psychiatry 1993;56:1169-1177)}

A current hypothesis about the specific role of the basal ganglia in motor control is that they are involved in motor programming. ${ }^{1} \mathrm{~A}$ number of studies have examined this hypothesis by assessing simple (SRT) and choice reaction time (CRT) in Parkinson's disease-for example, ${ }^{2-6}$. Despite the inconsistencies in the specific patterns of SRT and CRT deficit found across these studies, there is general agreement that patients with Parkinson's disease have longer reaction times than agematched normals. Such prolongation, however, is not specific to Parkinson's disease. A body of evidence suggests that slowness is present across a range of brain pathologies. Prolonged SRT or CRT, or both, compared with normal subjects has been reported for patients with epilepsy, ${ }^{7}$ Alzheimer's disease, ${ }^{8}$ and various cases of head injury. ${ }^{9}$ Slowing is also observed in psychiatric conditions such as depression and schizophrenia, ${ }^{10}$ as well as in normal ageing. ${ }^{11}$

From the available evidence, however, it could be argued that, although general slowing of reaction time may not be specific to Parkinson's disease, these patients exhibit a particular pattern of SRT/CRT impairment. A number of studies have found, relative to matched controls, that patients with Parkinson's disease are differentially or selectively impaired on SRT but not CRT tasks. ${ }^{2-5}$ This pattern of deficit has been interpreted as indicating that these patients fail to preprogramme- that is, fail to use the advance information about the nature of the response available to them in a SRT or precued CRT task to programme the response before the onset of the imperative stimulus. This pattern of deficits, however, does not have universal support-for example, ${ }^{3612}$. Furthermore, the inability to benefit from advance information in a precued CRT condition (taken as equivalent to SRT) has 
been reported for patients with frontal lobe damage, ${ }^{13}$ lesions of the supplementary motor area, ${ }^{14}$ and Alzheimer's disease ${ }^{8}$ and is not specific to Parkinson's disease.

Most published reports on reaction time in Parkinson's disease have been concerned with the issue of normality - that is, whether the pattern of reaction times and use of advance information in patients with Parkinson's disease differ from those of matched normal subjects. A second and important question that arises is one of specificity-that is, whether the particular pattern of deficits is specific to Parkinson's disease or is also present in other disorders of the basal ganglia. To date, only a handful of studies has addressed this question. In the study of Girotti et $a l,{ }^{15}$ Huntington's disease patients had significantly longer CRT compared with patients with Parkinson's disease. Halsband et $a l^{16}$ compared the performance of 14 patients with Huntington's disease, nine with Parkinson's disease, and 20 normal controls on two tasks, one requiring precise aiming movements and the other involving idiographic writing. On the aiming tasks, the patients with Parkinson's and Huntington's disease showed similar increases in movement time as a function of the difficulty of the task, relative to normal subjects. On the writing task, however, the patients with Huntington's disease showed disproportionate increases in writing time when letter size was increased. Viallet et $a l^{17}$ found that the SRTs of six patients with progressive supranuclear palsy were significantly longer than those of 13 patients with Parkinson's disease. Similarly, Dubois et al ${ }^{18}$ reported that 10 patients with progressive supranuclear palsy were significantly slower than the 33 patients with Parkinson's disease on SRT as well as go-no go CRT. The results of these studies suggest that reaction time deficits may not be specific to Parkinson's disease but also may be present in other forms of striatal pathology. The relatively simple paradigms used, however, offer little opportunity for determining the processes that underlie the slowness observed in the different groups.

The other aspect of the specificity question is whether the impairment of reaction time is specific to disorders of the basal ganglia or is also a feature of other movement disorders. It has been suggested that the lateral cerebellum $^{19}$ as well as the supplementary motor area ${ }^{20}$ play a role in motor programming. On this basis, it would be expected that patients with cerebellar disease or those with lesions of the supplementary motor area also would show deficits in reaction time that tapped this process. Some evidence for this exists. Nakamura and Taniguchi ${ }^{21}$ found that warned SRTs of 10 patients with cerebellar disease were significantly slower than those of 13 patients with Parkinson's disease and 14 normal subjects. Verfaellie and Heilman ${ }^{22}$ examined performance on uncued and precued CRT tasks in two patients with chronic, unilateral, medial frontal lobe lesions. The patient with a left-sided lesion benefitted from advanced information about the required response, whereas the patient with a right-sided lesion did not.

To date, no study has simultaneously addressed these two aspects of the specificity question: firstly whether the obtained patterns of reaction time deficit are specific to Parkinson's disease or are also present in other disorders of the basal ganglia; and secondly, whether they are also a feature of other movement disorders involving pathology outside the basal ganglia. The aim of the present study was to compare the pattern of performance of patients with Parkinson's, Huntington's and cerebellar disease.

The following questions were addressed. Firstly, how does the performance of patients with Parkinson's disease compare with that of patients with Huntington's or cerebellar disease on SRT and CRT tasks? Secondly, are there differences between the three groups in the ability to increase general alertness with a warning signal presented before the imperative stimulus? Thirdly, are there differences between the three groups in terms of their ability to develop an anticipatory set for the occurrence of the imperative stimulus and increase their general readiness to respond? Finally, are there differences between the three groups in the use of advance movement parameter information for preprogramming of motor responses?

\section{Method}

SUBJECTS

Three groups of patients took part in the study: eight patients with idiopathic Parkinson's disease, seven patients with Huntington's disease (all with a documented family history) and eight cases of idiopathic cerebellar degeneration with a late onset in most cases. Table 1 provides demographic and illness-related information of the groups.

All of the patients with Parkinson's disease were receiving dopaminergic medication (average levodopa dose $650 \mathrm{mg}$, SD $306 \mathrm{mg}$, with a peripheral decarboxylase inhibitor). In addition, two patients were on anticholinergics, three were taking a monoamine oxidase B inhibitor (selegeline/deprenyl), and three were taking amantadine. This group was tested in a drug-reduced state, after omitting the first morning dose of medication. The average time between taking the last dose of

Table 1 Details of patients with Parkinson's disease, Huntington's disease and cerebellar disease

\begin{tabular}{lccc}
\hline & $\begin{array}{l}\text { Parkinson's } \\
\text { disease }\end{array}$ & $\begin{array}{l}\text { Huntington's } \\
\text { disease }\end{array}$ & $\begin{array}{l}\text { cerebellar } \\
\text { disease }\end{array}$ \\
\hline Male & 5 & 4 & 5 \\
Female & 3 & 3 & 3 \\
Right handed & 6 & 6 & 8 \\
Left handed & 2 & 1 & 0 \\
MMSE score & $29 \cdot 1(1 \cdot 0)$ & $28 \cdot 6(1 \cdot 3)$ & $27 \cdot 3(2 \cdot 1)$ \\
BDI score & $6 \cdot 6(3.0)$ & $12 \cdot 1(6 \cdot 3)$ & $10 \cdot 3(4 \cdot 9)$ \\
Age & $61 \cdot 3(9 \cdot 7)$ & $44 \cdot 4(12 \cdot 9)$ & $55 \cdot 0(8 \cdot 0)$ \\
Age of onset & $53.0(10 \cdot 4)$ & $40 \cdot 3(11.4)$ & $44 \cdot 8(13.9)$ \\
Duration of illness & $8.3(5 \cdot 8)$ & $4 \cdot 1(2 \cdot 5)$ & $10.3(11 \cdot 3)$ \\
\hline
\end{tabular}

MMSE = mini-mental state examination; BDI = Beck depression inventory. Standard deviations are given in parentheses. 
medication on the previous evening and start of testing was $14 \cdot 4$ hours (SD $2 \cdot 2$, range 11-17 hours). One of the patients with Huntington's disease was taking haloperidol. None of the patients with cerebellar disease were taking any medication.

The mini-mental state examination (MMSE) ${ }^{23}$ and the Beck depression inventory $(\mathrm{BDI})^{24}$ were, respectively, used to screen for dementia and depression (table 1 gives the mean scores). A neurologist rated the patients with Parkinson's disease on the Webster scale $^{25}$ and those with Huntington's disease on the Marsden and Quinn chorea severity evaluation scale. ${ }^{26}$ The patients with cerebellar disease were assessed on an ataxia severity scale currently under development, with items relating to the performance of rapid alternating movements, dysmetria and intention tremor, sway and postural stability, gait, and speech. Scores on this scale range from 0 to 32 , with higher scores indicating more severe disease. The mean scores of the patients with Parkinson's disease on the Webster scale were 14.6 (SD 5.6). On medication, five of these were in Hoehn and $\mathrm{Yahr}^{27}$ stage III, one was in stage I and two were in stage II. The patients with Huntington's disease had mean chorea severity scores of 10.6 (SD 7.9). The average ataxia rating for the patients with cerebellar disease was 13.6 (SD $5 \cdot 2$ ). Although precise matching of patients with different disorders in terms of illness severity is not possible, nevertheless the ratings suggested that patients in the three groups were manifesting mild to moderately severe symptoms of their disease.

\section{REACTION TIME TASKS}

The response board had six circular buttons (diameter $2.5 \mathrm{~cm}$ ). Two black central buttons, $15 \mathrm{~cm}$ apart were the 'home' keys.

$\begin{array}{ccc}\begin{array}{c}\text { Fixation } \\ \text { point }\end{array} & \begin{array}{c}\text { Precue/warning } \\ \text { signal (S1) } \\ \text { (S1) }\end{array} & \begin{array}{c}\text { Imperative } \\ \text { stimulus (S2) }\end{array} \\ & 1-4 \mathrm{~s} & \begin{array}{l}0,200,800,1600, \\ 3200 \mathrm{~ms}\end{array}\end{array}$

Simple RT
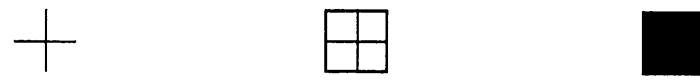

Uncued CRT
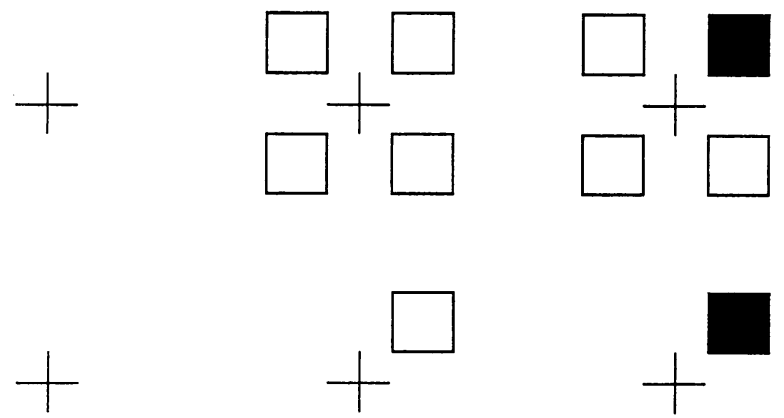

Figure 1 A diagrammatic representation of the procedure and the various experimental tasks.
There were two red response buttons $10 \mathrm{~cm}$ above these, and at the same distance below, two green response buttons.

Figure 1 shows the various reaction time tasks. The subject sat in front of a visual display unit and held down one or both of the "home" keys with the index finger of the right, left or both hands, depending on the condition. This made a small fixation cross appear in the middle of the screen. On $80 \%$ of the trials, after a variable delay (1-4 s), a precue/warning signal (S1) was presented. In all conditions, $\mathrm{S} 1$ was one or more empty squares. The imperative stimulus (S2) was the filling (becoming white on the black display) of the relevant square after an experimentally manipulated interval of 200,800 , 1600 or $3200 \mathrm{~ms}$. On $20 \%$ of trials, no S1 was given, the subject receiving only the imperative stimulus (unwarned/uncued trials). The subject's task was to respond as quickly as possible to the imperative stimulus by releasing the "home" key (reaction time) and moving to and pressing the relevant "response" key (movement time). The screen cleared $500 \mathrm{~ms}$ after a response was made, and the next trial was started when the subject returned to and depressed the "home" key. Anticipations (reaction times of $100 \mathrm{~ms}$ or less), long responses (over $3 \mathrm{~s}$ ), and decision errors (wrong response key pressed in CRT) were eliminated and the trial replaced.

\section{Simple reaction time}

In the SRT task each block of trials involved the same response-for example, moving the right index finger from the "home" key to the right upper "response" key-to the same stimulus. Two versions of the SRT task were used: a "random block" SRT and a "fixed block" SRT condition. In the "random block" condition, trials with each of the five S1-S2 intervals were randomly mixed, with 10 trials per interval. In the "fixed block" SRT condition, 10 consecutive trials were given for each of the S1-S2 intervals. The order of the five S1-S2 intervals was counterbalanced within each group. All subjects were tested separately with right and left hands on the two SRT tasks. The order of testing for hand and condition was counterbalanced within each group.

\section{Four choice reaction time}

The two movement parameters involved were hand (right versus left) and direction (up versus down). The response was fully cued or uncued across the conditions. Each CRT condition consisted of a block of 75 trials, with 15 trials at each of the five S1-S2 intervals randomly mixed. An approximately equal and randomly mixed number of right and left hand responses were incorporated. In the uncued task (see fig 1), four empty squares appeared on the display to the left and right and above and below the fixation point. After the S1-S2 interval, one of the four squares filled, indicating the response button to be pressed. In the fully cued condition (see fig 1), a single empty square appeared in one of 
the four possible positions above or below, to the left or right of the fixation point, its spatial location providing complete and precise information about the required response to the subject. After the S1-S2 interval, the square filled, instructing the subject to move to the appropriate key. All subjects performed the SRT conditions first, followed by the CRT conditions. Within each group, the order of the CRT conditions was counterbalanced. To assess practice or fatigue effects, the random block SRT condition was repeated at the end of the session.

\section{STATISTICS}

The $\chi^{2}$ test was used for examining distribution of variables (sex, handedness) across the three groups. With normally distributed data, group differences were analysed using analysis of variance. When the data were not normally distributed, non-parametric Kruskal-Wallis analyses of variance were used.

\section{Results}

Table 1 lists the demographic and illnessrelated details of the groups. There were no differences across the groups in the distribution of men and women or in terms of handedness $(p>0.05)$. The differences in age between the groups were significant $(p<0.05)$, with the patients with Parkinson's disease being older than those with Huntington's disease $(p<0.05)$. The three patient groups did not differ in terms of duration of illness or mean age of onset of the disease $(p>0.05)$. While the differences in the MMSE scores approached significance $(p=$ 0.07 ), none of the patients had MMSE scores lower than the cut off of $23 .{ }^{23}$ The groups did not differ significantly in mean scores on the BDI ( $p>0.05$ ), and two of the patients with Huntington's disease and one of those with cerebellar disease had BDI scores above the cut off of 17 used for differentiating moderate to severe depression. ${ }^{24}$

Comparing overall mean SRT at the beginning and end of the session, showed a mean increase of $65 \mathrm{~ms}$ for the patients with Parkinson's disease, $10 \mathrm{~ms}$ for those with cerebellar disease, and $34 \mathrm{~ms}$ for those with Huntington's disease. The effect of test occasion was significant $(p<0.01)$, but not the group, or the group by test occasion interaction $(p>0.05)$. In all three groups SRTs increased, indicating a similar, within-session fatigue effect.

As the patients with Parkinson's and Huntington's disease differed in age, and group differences in MMSE scores approached significance, all analyses were repeated using age and MMSE scores as covariates. Regression statistics showed that age and MMSE were not significant covariates, however, and the results did not differ when these covariates were or were not included. The results of the simpler analysis of variance without covariates are, therefore, reported. In all analyses of variance, group

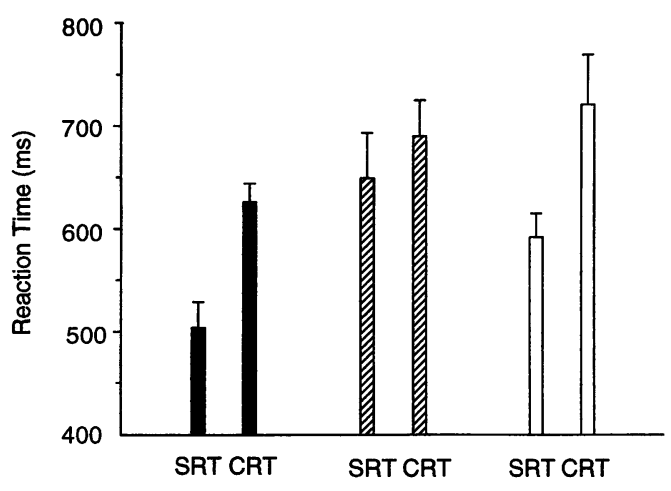

Figure 2 The mean unwarned (no S1) simple reaction time (SRT) and the unwarned and uncued choice reaction time (CRT) conditions of the patients with Parkinson's disease ( $\mathrm{\square})$, Huntington's disease $(\mathbb{Q})$ and cerebellar disease ( $\square$ ).

was the between groups factor and reaction time condition with or without S1-S2 interval were the within-subject, repeated measures factors.

\section{UNWARNED AND UNCUED SRT AND CRT}

To compare true SRT and CRT, the trials without an S1 from the random block SRT and the uncued CRT tasks were compared. Figure 2 shows the data for the three groups. The groups differed in SRT $(p<0.01)$. Post hoc analysis using the Scheffe test revealed that this resulted from the SRT of the patients with Huntington's disease being significantly longer than that of the patients with Parkinson's disease $(p<0.05)$, whereas the differences in SRT between the patients with cerebellar disease and the other two groups were not significant. The three groups did not differ in terms of CRT ( $p>0.05$ ). Paired student's $t$ tests were used to analyse SRT/CRT differences within each group. CRT was significantly longer than SRT for the patients with Parkinson's disease (121 ms difference) $(p<0.01)$ and for those with cerebellar disease $(129 \mathrm{~ms}$ difference $)(\mathrm{p}<0.05)$. For the

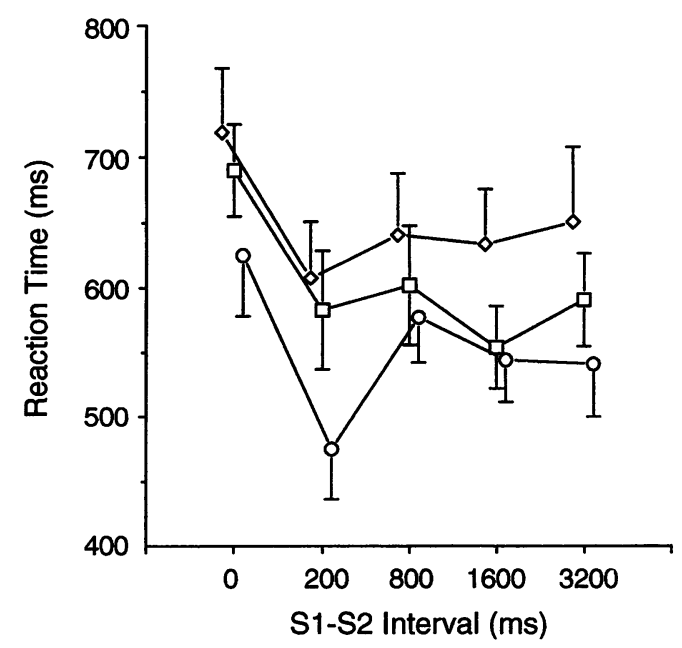

Figure 3 The mean reaction times of the patients with Parkinson's disease (O), Huntington's disease $(\square)$ and cerebellar disease $(\diamond)$ across the five S1-S2 intervals in the uncued choice reaction time condition. 
patients with Huntington's disease, however, the difference between CRT and SRT (41 ms) was not significant ( $p>0.05)$.

EFFECTS OF A WARNING SIGNAL

The effects of a warning signal-that is, a stimulus having a general alerting effect without providing specific information about the particular response required-was assessed in the uncued CRT condition by comparing reaction times for the unwarned (S1-S2 interval of $0 \mathrm{~ms}$ ) with the mean of the warned trials (S1-S2 interval of 200,800, 1600 or $3200 \mathrm{~ms}$ ). Figure 3 shows the data. Presence of a warning signal significantly decreased reaction times for all three groups ( $p<$ $0.001)$. The three groups did not differ ( $p>0.05$ ). The group by warned/unwarned

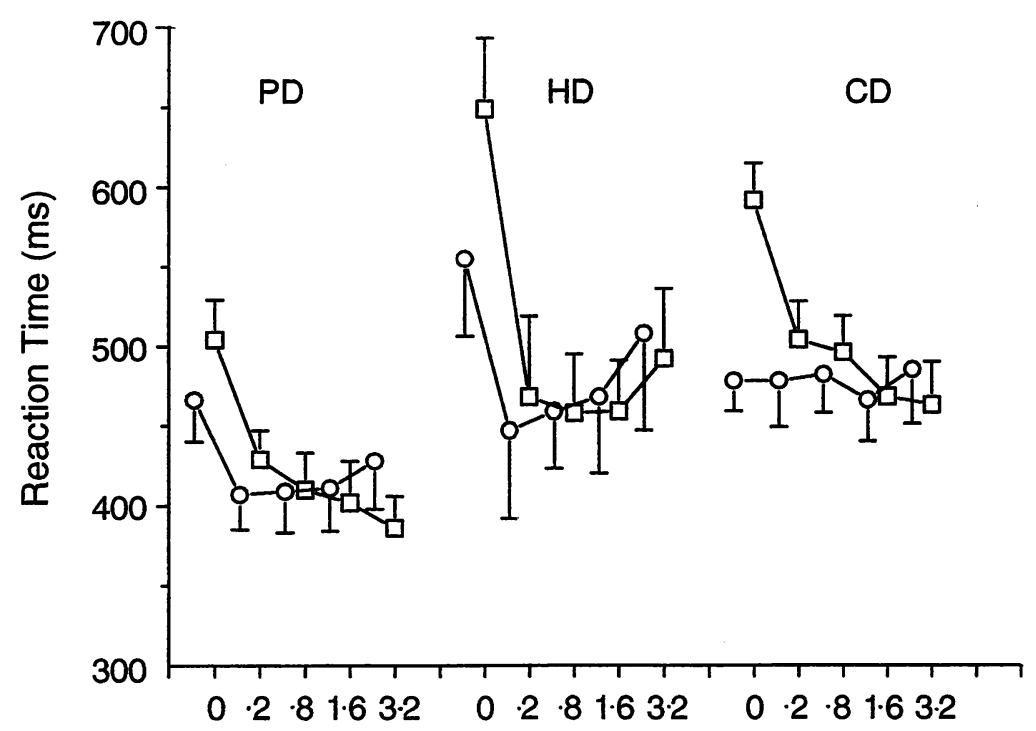

Figure 4 The mean reaction times of the patients with Parkinson's disease (PD), Huntington's disease (HD), and cerebellar disease (CD) across the five S1-S2 intervals in the fixed block $(\bigcirc)$ and random block $(\square)$ simple reaction time conditions.

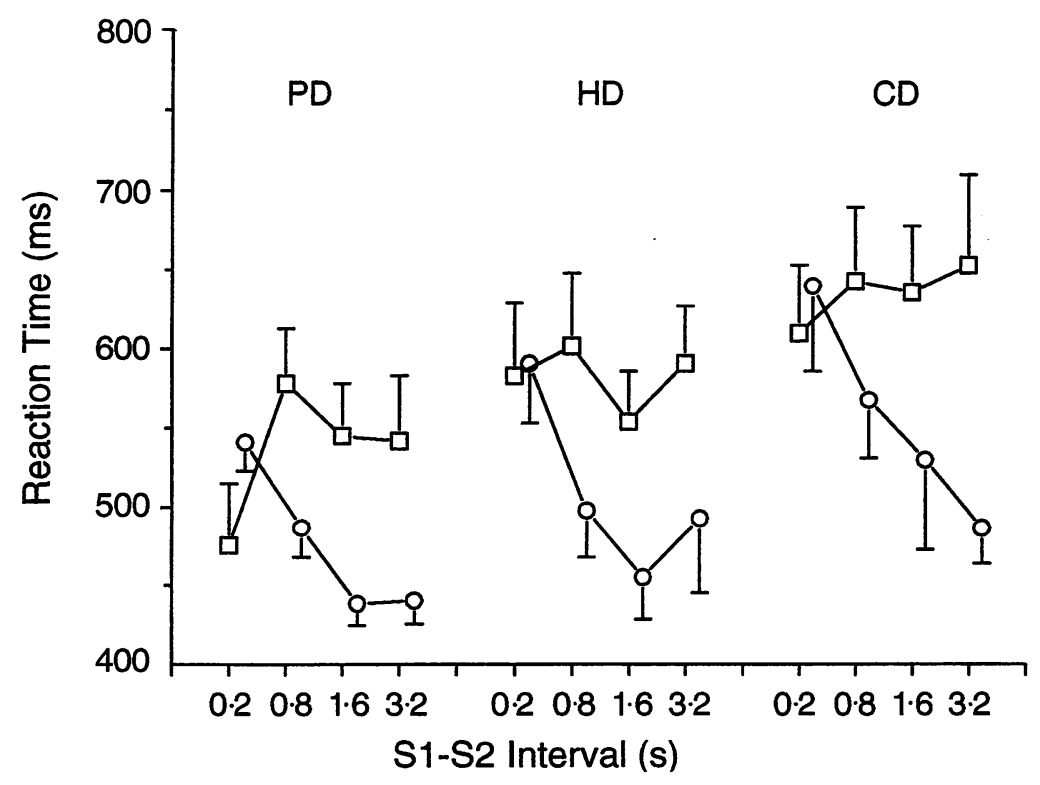

Figure 5 The mean reaction times of the patients with Parkinson's disease (PD), Huntington's disease (HD) and cerebellar disease (CD) across the 200 to $3200 \mathrm{~ms} S 1-\mathrm{S} 2$ intervals in the uncued $(\square)$ and fully cued $(O)$ choice reaction time conditions. condition interaction was not significant $(p>$ 0.05). The pattern of shortening of CRT with a warning signal in the three groups was examined further by comparing performance across the four warning signal intervals (200, $800,1600,3200 \mathrm{~ms})$. The patients with Parkinson's disease showed an enhanced warning signal effect with an S1-S2 interval of $200 \mathrm{~ms}$ relative to the other intervals $(p<0.05)$. In contrast, the other groups showed no significant difference in RT across the four, warned intervals (see fig 3 ).

\section{EFFECTS OF TEMPORAL EXPECTANCY}

The effects of temporal expectancy were assessed by comparing the random and fixed block SRT (the latter provided the opportunity for development of an anticipatory set for the onset of the imperative stimulus in the warned trials). In the unwarned trials, the absence of a warning signal is also predictable in the fixed block task. The data are shown in fig 4. Of all the main and interaction effects, the significant ones were the main effect of interval $(0,200,800,1600,3200 \mathrm{~ms})$ $(p<0.001)$, the Group by interval interaction $(\mathrm{p}<0.01)$, and the condition (fixed vs random block) by interval interaction $(p<0.001)$. The group by interval interaction was explored further by examining the main effect of interval in each of the two SRT conditions and in each group separately. For the patients with Parkinson's disease and Huntingdon's disease, the main effect of interval was significant in both the fixed and random block SRT conditions $(p<0.001)$.

For the cerebellar patients, however, the main effect of interval was significant in the random block SRT $(p<0.001)$, but not in the fixed block SRT condition $(p>0.05)$. In relation to the significant condition by interval interaction, further post hoc analyses revealed that, for the patients with Huntington's disease $(p<0.01)$ and those with cerebellar disease $(p<0.01)$, reaction times for the unwarned trials (S1-S2-interval of $0 \mathrm{~ms}$ ) were significantly faster in the fixed block than in the random block condition. For the patients with Parkinson's disease, this difference was not significant $(p>0.05)$.

USE OF ADVANCE SPATIAL INFORMATION

Group differences in the use of full advance information about the required response compared with the uncued CRT condition were examined for the 200 to $3200 \mathrm{~ms} \mathrm{S1-S2}$ intervals (fig 5). There were no differences between the groups $(p>0.05)$. The main effects of condition and interval and their interaction were significant $(p<0.001)$. In contrast, none of the interactions involving group were significant $(p>0.05)$. Across the three groups, the significant main effect of condition resulted from reaction times in the uncued CRT being slower than those in the fully cued CRT task $(p<0.001)$. From this it can be inferred that patients in all three groups used the advance information provided by the precue, to preprogramme the 
Table 2 Mean (SD) of movement time in each of the conditions for the patients with Parkinson's, Huntington's and cerebellar disease

\begin{tabular}{llll}
\hline & \multicolumn{1}{l}{ Parkinson's disease (ms) } & Huntington's disease (ms) & Cerebellar disease (ms) \\
\hline Simple RT & $389(65)$ & $453(165)$ & $523(126)$ \\
Choice RT & $469(84)$ & $480(176)$ & $658(88)$ \\
Uncued & $499(79)$ & $503(215)$ & $709(174)$ \\
Fully cued &
\end{tabular}

Mean movement times are the averages of the five S1-S2 intervals. $\mathrm{RT}=$ reaction time.
ERRORS

Very few errors were made in any of the groups. The groups differed significantly in terms of anticipations in the random block SRT (mean errors Parkinson's: 0.75; Huntington's: 4.0; cerebellar: $1.1 ; p<0.01$ ) and the fully-cued CRT (mean errors Parkinson's: $0 \cdot 43$; Huntington's: $12 \cdot 8$; cerebellar: $6.1 ; \mathrm{p}<0.05)$ conditions. Post hoc analyses revealed that the above group differences in SRT resulted from the patients with Huntington's disease having significantly ( $p<$ $0 \cdot 50$ ) more anticipations than the other two groups, with no differences between the latter groups. In the precued CRT, the patients with Parkinson's disease had significantly more anticipations than the Huntington's disease and cerebellar disease cases $(p<0.05)$, whereas the differences between the other two groups did not attain significance. , suggests that longer prep intervals allowed subjects to benefit more from the advance information provided by $\mathrm{S} 1$. In contrast, in the uncued CRT condition, longer S1-S2 intervals produced no beneficial effects on reaction time beyond that evident even at $200 \mathrm{~ms}$ as a result of the presence of a warning signal.

\section{MOVEMENT TIME}

Table 2 shows the mean movement times in the SRT and CRT conditions. With regard to group differences in movement time between the SRT and CRT tasks, the main effect of group $(p<0.05)$ and condition $(p<0.001)$ were significant, whereas their interaction was not $(p>0.05)$. The significant condition effect arose from longer movement times for the CRT than the SRT task. The group effect resulted from the movement times of the patients with cerebellar disease being longer than those of patients with Huntington's and Parkinson's disease in both the SRT and CRT conditions $(p<0.05)$. Across all conditions, movement times of the patients with Huntington's disease tended to be slower than those with Parkinson's disease, although none of the differences were significant $(p>$ $0.05)$. Group differences in movement time between the two CRT conditions were also examined. The main effects of group and condition $\quad(p<0.05)$ were significant, whereas their interaction was not $(p>0.05)$. As is evident from table 2 , the main effect of condition resulted from movement time in the precued CRT being slower than that in the uncued CRT task in all three groups (the difference only being significant for the patients with Parkinson's disease). Post hoc analyses revealed that, across the conditions, the main effect of group resulted from movement times of the patients with cerebellar disease being longer than those with Parkinson's disease and Huntington's disease $(p<0.05$, except for comparison of the groups with Huntington's and cerebellar disease in precued CRT condition $p<0.06)$. Although the movement times of the patients with Parkinson's disease were faster than those with Huntington's disease, the differences were not significant $(p>0 \cdot 05)$.

\section{Discussion}

Before discussing the implications of the results, the effect of a number of factors that could potentially confound the findings need to be considered. Although the patients with Huntington's disease were younger and had lower MMSE scores than the Parkinson's disease patients, neither age nor MMSE were significant covariates for the reaction time measures and their inclusion as covariates did not alter the pattern of results. A significant fatigue effect, as indexed by a slowing of SRT from the begining to the end of the session, was present in all three groups. Therefore, the pattern of between group results was not confounded by differential fatigue effects. In all three groups, very few errors of any type were made. The only group differences in error was in terms of the anticipations in the SRT and fully cued CRT conditions. In any case, the reaction time data analysed were based on error-free trials.

\section{GROUP DIFFERENCES IN ALERTNESS AND} MOTOR READINESS

With regard to the beneficial effects of a warning signal, in all three patient groups, CRTs were reduced when a warning signal was present compared with the unwarned trials (fig 3). Furthermore, for the patients with Parkinson's disease, the speeding of CRT with a warning signal was maximal when a warning signal was presented $200 \mathrm{~ms}$ before the imperative stimulus. This optimal period of $200 \mathrm{~ms}$ for a warning signal in CRT is typically found in normal subjects. ${ }^{628}$ This pattern was not found in the other two groups.

In all three patient groups, the presentation of a warning signal also reduced reaction times compared with unwarned trials in the random block SRT (fig 4). This reduction with a warning signal was also evident in the fixed block SRT condition for the groups with Parkinson's and Huntington's, but not the patients with cerebellar disease.

In normal subjects, the use of a fixed S1-S2 interval or preparatory interval reduces 
reaction times. ${ }^{29-30}$ In the present study, as in our previous studies, ${ }^{631}$ however, no differences in reaction time between random and fixed block SRT conditions were observed, as the relatively small number of trials at each preparatory interval may have been insufficient to promote the development of an anticipatory set and augment motor readiness beyond that achieved by the use of warning signals in the random block SRT. The fixed block SRT condition in fact employed two types of predictability. The first was the temporal predictability of $\mathrm{S} 2$ following $\mathrm{S} 1$ in trials where a warning signal was given (200-3200 ms). The second aspect of predictability was for those trials where no warning signal was given. In this case the subject could benefit from the knowledge that the trial would be unwarned only if he or she was able or willing to maintain a constant state of response readiness during the period of time following the last response to the onset of the imperative stimulus. The results from the present study suggest that the three groups differed in this aspect of predictability. The absence of a warning signal and interval effect in the fixed block condition for the patients with cerebellar disease suggests that, in this condition, they were able to maintain a general motor readiness even without a warning signal. The performance of the patients with Huntington's disease was intermediate. The fact that reaction times for the unwarned trials were significantly faster in the context of the fixed block SRT than the random block SRT, suggested that the patients with Huntington's disease could maintain motor readiness to some extent, but not as well as the patients with cerebellar disease who, in addition, did not show any interval effect in the fixed block SRT condition. The patients with Parkinson's disease when tested off medication, however, needed the external trigger of a warning signal to generate a state of readiness to respond when the onset of the imperative stimulus was not temporally predictable from the warning signal. This apparent over-reliance of the patients with Parkinson's disease on a warning signal in order to maintain motor readiness is consistent with the finding that provision of external cues improved the ability of these patients to maintain motor set, ${ }^{32}$ and the suggestion that these patients have difficulty in internal but not external control of attention. ${ }^{33}$

To summarise: firstly, patients in all three groups benefited from a warning signal; secondly, for the patients with Parkinson's disease, the reduction of CRT was maximal with a warning signal presented $200 \mathrm{~ms}$ before the imperative stimulus; and finally, in the context of the predictable absence of a warning signal in SRT, the patients with cerebellar disease and to a lesser extent those with Huntington's disease could maintain motor readiness. In contrast, the patients with Parkinson's disease tested off medication required the external trigger of a warning signal to generate and maintain a state of motor readiness.
GROUP DIFFERENCES IN SRT AND CRT AND THE USE OF ADVANCE INFORMATION

Before discussing the reaction time data, it is necessary to consider movement time, to ensure that the effects of advance information are only reflected in the first and not the second sets of data. In the SRT condition, where the same discrete movement to a single target was involved across trials, movement times were faster in all three groups than in the CRT conditions, where the position of the target changed across trials. The important thing to note, however, is that since movement times were in fact slower in the precued condition relative to the uncued CRT, provision of advance spatial information reduced reaction but not movement times. The patients with cerebellar disease had significantly longer movement times than the patients with Huntington's disease and those with Parkinson's disease across the SRT and CRT conditions. Movement times of the patients with Huntington's disease were, in turn, slower than those with Parkinson's disease across the SRT and CRT conditions, although none of the differences were significant. As noted above, however, because there is no evidence that provision of advance spatial information reduced movement times, no differences in motor programming can be inferred from these group differences.

For the patients with Parkinson's disease or cerebellar disease, uncued CRT was significantly slower than SRT. For the Huntington's disease patients, however, SRT did not differ from CRT. This suggests that the patients with Huntington's disease were failing to engage in the optional preprogramming which is essential for conferring its speed advantage to SRT. However, in the fully cued CRT task, advance information was used by all three patient groups to preprogramme responses, as indicated by faster reaction times in this condition relative to the uncued CRT. Therefore, in contrast to SRT, the process of programming a response when performed before the imperative stimulus on the basis of explicit, external advance spatial information (precued CRT task) or after the imperative stimulus (uncued CRT) did not reveal any differences between the patients with Parkinson's, Huntington's or cerebellar disease.

How can this failure of the patients with Huntington's disease to preprogramme the response in SRT be explained? One reason may be that preprogramming in SRT is an optional, volitional and resource-demanding strategy. As noted by Klapp ${ }^{34}$ : 'Such advance programming would not be obligatory when the SRT paradigm is used since subjects could merely ignore the advance information and postpone programming until the onset of the "go" signal'. (pp.234-5). In addition, in SRT, as programming of the response occurs before the stimulus, it is volitionally (internally) controlled rather than externally triggered. ${ }^{35}$ Results of studies examining the degree of interference produced by a secondary task on SRT and CRT, ${ }^{53}$ have shown 
that the volitional control necessary for preprogramming of the response in SRT is a resource-demanding process. The failure to preprogramme the response in SRT by the group with Huntington's disease can be interpreted as indicating an inability to engage in volitional control of action, possibly because of its resource-demanding nature. Whether this is due to a reduction of processing resources or less efficient use of available resources has not been explored. The availability and strategic allocation of processing resources in Huntington's disease needs to be addressed in the future.

The only quantitative group difference in reaction time was in the patients with Huntington's disease, who had a significantly slower SRT than the group with Parkinson's disease. The groups did not differ with regard to uncued CRT. With the exception of the unwarned SRT task, across conditions, patients with Parkinson's disease had the fastest and the cerebellar group had the slowest reaction times, with the Huntington's disease patients falling in between, although the differences were not significant. In previous comparative studies, patients with cerebellar disease $^{21}$ or Huntington's disease ${ }^{15}$ have been found to have significantly longer SRTs or CRTs than those with Parkinson's disease. The apparent discrepancy between the present results and those of the previous studies may be related to two factors. Firstly, the specific nature of the tasks used differed across studies. For example, Nakamura and Taniguchi's ${ }^{21}$ SRT task involved bimanual flexion or supination of the forearms to an auditory signal. Furthermore, premotor-that is, the interval between go signal and EMG onset-SRT was measured. The type of response and its method of measurement are different therefore, compared with the present study which involved a single finger lifting total SRT (interval between go signal and onset of behavioural response-that is, premotor and motor RT). Differences in the nature of the stimuli and responses are also evident between the uncued CRT task of this study, which required lifting either the right or left hand from one of two "home" keys, and movement in the vertical plane to one of four upper or lower "response" keys when the go signal was visually presented on a display, and that of Giortti et $a l^{15}$ which involved the movement of the finger from a single central

Table 3 A summary of the similarities and differences in the reaction time performance of patients with Parkinson's, Huntington's and cerebellar disease

\begin{tabular}{|c|c|c|c|}
\hline & $\begin{array}{l}\text { Parkinson's } \\
\text { disease }\end{array}$ & $\begin{array}{l}\text { Huntington's } \\
\text { disease }\end{array}$ & $\begin{array}{l}\text { Cerebellar } \\
\text { disease }\end{array}$ \\
\hline $\begin{array}{l}\text { Able to make use of a warning signal to reduce RT } \\
\text { relative to unwarned trials in an uncued CRT task }\end{array}$ & Yes & Yes & Yes \\
\hline $\begin{array}{l}\text { Able to maintain state of motor readiness on trials } \\
\text { when it is known that there will be no warning signal } \\
\text { (0 ms S1-S2 interval in fixed block SRT task) }\end{array}$ & No & Yes/No & Yes \\
\hline $\begin{array}{l}\text { Able to make use of response invariance in SRT } \\
\text { to reduce RT relative to CRT task }\end{array}$ & Yes & No & Yes \\
\hline $\begin{array}{l}\text { Able to make use of advance spatial information } \\
\text { to reduce RT in a precued CRT task relative to } \\
\text { uncued CRT }\end{array}$ & Yes & Yes & Yes \\
\hline
\end{tabular}

$\mathrm{RT}=$ reaction time; $\mathrm{CRT}=$ choice reaction time; $\mathrm{SRT}=$ simple reaction time. "home" key to one of several peripheral "response" keys when these lit, thus necessitating lateral movements in the horizontal plane.

The second factor that may account for the apparent discrepancy in the statistical significance of group differences across various studies is the issue of variability. Whether differences between groups attain statistical significance or not across studies depends on such factors as sample size, the number of reaction time trials, or the amount of practice. These are likely to affect reaction time variability, and ultimately determine the degree of within group variability compared with between groups variability. Therefore, regardless of whether any quantitative differences in reaction time between the groups attained statistical significance or not, an important consideration is whether the pattern of results are qualitatively similar or different in the three patient groups. Table 3 summarises the similarities and differences between the three patient groups across the various experimental conditions of the present study.

Firstly, we will consider group similarities and differences in alertness and motor readiness. Presentation of a warning signal speeded up CRT and randomised SRT in all groups, indicating that the groups did not differ in terms of general alertness. Qualitatively, the only difference between groups was that, with prior knowledge that trials would be unwarned (fixed block SRT), patients with cerebellar disease and, to a lesser extent, those with Huntington's disease, were able to maintain motor readiness even in the absence of a warning signal, whereas those with Parkinson's disease, tested off medication, were unable to do so. As noted above, these deficits in maintaining motor readiness without a warning signal in patients with Parkinson's disease may relate to the overreliance of these patients on the external control of attention.

Secondly, the pattern of SRT/CRT performance and the use of advance information suggests that the processes of stimulus identification, response selection, and response programming are quantitatively as well as qualitatively similar in the patients with Parkinson's disease and cerebellar disease. The patients with Huntington's disease appeared able to use advance information in the precued CRT task but did not engage in advance programming of the invariant response when this was optional in the SRT task.

In the present study, one simple interpretation of this general similarity in the reaction time performance of those with Parkinson's cerebellar and to a lesser extent, Huntington's disease, is that these deficits are a non-specific concomitant of any form of brain damage. Such a suggestion is consistent with a body of evidence referred to earlier. Therefore slowness in RT is present across a range of brain pathologies. What is, in effect, observed across a range of disorders affecting 
brain function, including the three patient groups in the present study, is behavioural slowness. The important question relates to the mechanism(s) underlying this slowness in the different diseases. One possible explanation is that brain damage of any sort may affect some global or general function, such as attentional processing or motor readiness, which is manifested as behavioural slowness.

The second possibility is that the observed behavioural slowness in the different disorders is in fact mediated by different mechanisms. The motor symptomatology and pathophysiology of Parkinson's, Huntington's and cerebellar disease differ in fundamental ways. In Parkinson's disease, during ballistic movements, the size of the first agonist burst is reduced, thus requiring additional cycles of agonist/antagonist activity for completion of a movement. ${ }^{36}$ In contrast, in cerebellar disease, the initial bursts of activity in the agonist or antagonist muscles, or both, are prolonged and the timing of antagonist inhibition is abnormal, which has respectively been noted to underlie dysmetria and dysdiadochokinesia. ${ }^{37}$ In patients with Huntington's disease, Hefter et al ${ }^{38}$ found a deficit in building up EMG activity, which caused prolongation of EMG bursts and slowness of contraction. Although there is diverse pathology in each of the three diseases, it may be that the critical pathology differs in these disorders and involves the cerebellum in cerebellar disease, the putamen in Parkinson's disease, and the caudate in Huntington's disease. Each of these regions, in turn, projects predominantly via distinct parts of the thalamus to discrete areas of frontal cortex: motor and premotor cortex from the cerebellum; the supplementary motor area from the putamen; and prefrontal cortex from the caudate. The central mechanisms of behavioural slowness may be different in Parkinson's, Huntington's, and cerebellar disease, therefore, and the unravelling of the mechanisms of motor slowness in these movement disorders remains a task for future studies.

This work was supported by a grant from the Wellcome Trust. We would like to thank Professor Anita Harding and Dr Niall Quinn for allowing us to see patients in their care. We would also like to thank Drs Tom Britton, Peter Brown and Malcolm Steiger for the clinical assessment of the patients.

1 Marsden CD. The mysterious motor function of the basal ganglia. The Robert Wartenberg Lecture. Neurology 1982;32:514-39.

2 Bloxham CA, Mindell TA, Frith CD. Initiation and execution of predictable and unpredictable movements in Parkinson's disease. Brain 1984;107:371-84

3 Stelmach GE, Worringham CJ, Strand EA. Movement preparation in Parkinson's disease. The use of advance information. Brain 1986;109:1179-94.

4 Pullman SL, Watts RL, Juncos JL, Chase TN, Sanes JN. Dopaminergic effects on simple and choice reaction time performance in Parkinson's disease. Neurology 1988;38:249-54

5 Goodrich S, Henderson I Kennard C. On the existence of an attention-demanding process peculiar to simple reaction time: Converging evidence from Parkinson's disease. Cogn Neuropsychol 1989;6:309-31.

6 Jahanshahi M, Brown RG, Marsden CD. Simple and choice reaction time and the use of advance information for motor preparation in Parkinson's disease. Brain 1992;115:539-64.

7 Bruhn P, Parsons OA. Reaction time variability in epileptic and brain-damaged patients. Cortex 1977;13:373-84.

8 Gordon B, Carson K. The basis for choice reaction time slowing in Alzheimer's disease. Brain Cogn 1990;13: 148-66.

9 Miller E. Simple and choice reaction time following severe head injury. Cortex 1976;6:121-7.

10 King HE. Psychomotor aspects of mental disease. Harvard University Press: Cambridge, 1954

11 Wilkinson RT, Allison S. Age and simple reaction time: Decade differences for 5,325 subjects. F Gerontol Psychol Sci 1989;44:29-35.

12 Mayeux R, Stern Y, Sano M, Cote L, Williams JBW Clinical and biochemical correlates of bradyphrenia in Parkinson's disease. Neurology 1987;37:1130-4.

13 Alivisatos B, Milner B. Effects of frontal or temporal lobectomy on the use of advance information in a choice reaction time task. Neuropsychologia 1989;27:495-503.

14 Verfaellie M, Heilman KM. Response preparation and response inhibition after lesions of the medial frontal response inhibition after lesions of
lobe. Arch Neurol 1987;44:1265-71.

15 Girotti F, Marano R, Soliveri G, Geminiani G, Scigliano G. Relationship between motor and cognitive disorders G. Relationship between motor and cognitive disord
in Huntington's disease. $\mathcal{F}$ Neurol 1988;235:454-7.

16 Halsband U, Homberg V, Lange HJ. Slowing of different types of voluntary movement in extrapyramidal disease: Fitt's law and idiographic writing. In: Beradelli A Benecke R, Manfredi M, Marsden CD, eds. Motor disturbances II, London, Academic Press: 1990;1 181-90

17 Viallet F, Trouche E, Beaubaton D, Legallet E, Khalil R. Visual feedback and motor performance in human and animal basal ganglia dysfunction. In: Schneider JS, Libby TI, Eds. Basal ganglia and behavior, Toronto: Huber, 1987;71-82.

18 Dubois B, Pillon B, Legault F, Agid Y, Lhermite F Slowing of cognitive processing in progressive supranuSlowing of cognitive processing in progr
clear palsy. Arch Neurol 1988;45:1194-9.

19 Allen GI, Tsukahara N. Cerebrocerebellar communication systems. Physiol Rev 1974;54:957-1006.

20 Evarts EV, Wise SP. Basal ganglia and motor control. In Evered D, O'Connor M, eds. Functions of the basal ganglia: Ciba Foundation symposium 1984;107:83-96.

21 Nakamura R, Taniguchi R. Dependence of reaction times on movement patterns in patients with Parkinson's disease and those with cerebellar degeneration. Tohoku Exp Med 1980;132:153-8.

22 Verfaellie M, Heilman KM. Response preparation and response inhibition after lesions of the medial frontal response inhibition after lesions
lobe. Arch Neurol 44:1265-71.

23 Folstein MF, Folstein SE, McHugh PR “Mini-Mental State": A practical method for grading the cognitive State": A practical method for grading the cognitive
state of patients for the clinician. $\mathcal{F}$ Psychiatr Res 1975 ; state of patien
12:189-98.

24 Beck AT, Ward CH, Mendelson M, Mock JE, Erbaugh JK. An inventory for measuring depression. Arch Gen Psychiatry 1961;4:561-71.

25 Webster DD. Clinical analysis of the disability in Parkinson's disease. Mod Treat 1968;5:257-82.

26 Marsden CD, Quinn N. Chorea Severity Evaluation Scale. In: Marsden CD, Schachter M, Assessment of extrapyramidal disorders. $\mathrm{Br} \mathcal{F}$ Pharmacol 1981;11: 129-51.

27 Hoehn MM, Yahr MD. Parkinsonism: onset, progression and mortality. Neurology 1967;17:427-42.

28 Posner MI, Klein R, Aummers J, Buggie SI. On the selection of signals. Memory Cogn 1973;1:2-12.

29 Zahn TP, Rosenthal D. Simple reaction time as a function of the relative frequency of the preparatory interval. f Exp Psychol 1966;72:15-9.

30 Granjon MJ, Requin J, Durup H, Reynard G. Effects of a timing signal on reaction time-time with 'non-aging' foreperiod. $\mathcal{F}$ Exp Psychol 1973;101:139-45.

31 Jahanshahi M, Brown RG, Marsden CD. The effect of withdrawal of dopaminergic medication on simple and choice reaction time and the use of advance information for motor preparation in Parkinson's disease. $\mathcal{f}$ Neurol Neurosurg Psychiatry 1992;55:1168-76.

32 Robertson C, Flowers KA. Motor set in Parkinson's disease. ₹ Neurol Neurosurg Psychiatry 1990;53:583-92.

33 Brown RG, Marsden CD. Internal versus external cues and the control of attention in Parkinson's disease. and the control of atten

34 Klapp ST. Reaction time analysis of programmed control. Exerc Sport Sci Rev 1977;5:231-53.

35 Frith CD, Done DJ. Routes of action in reaction time tasks. Psychol Res 1986;48:169-77.

36 Hallett M, Khoshbin S. A physiological mechanism of bradykinesia. Brain 1980;103:301-14

37 Hallett M, Shahani BT, Young RR. EMG analysis of patients with cerebellar deficits. If Neurol Neurosurg Psychiatry 1975;38:1163-9.

38 Hefter H, Homberg V, Lange HW, Freund H-J. Impairment of rapid movement in Huntington's disease. Brain 1987;110:585-612. 